\title{
Detection of Solvents using a Distributed Fibre Optic Sensor
}

\author{
Alistair MacLean, Chris Moran, Walter Johnstone, Brian Culshaw, Dan Marsh, Paul \\ Parker.
}

A fibre optic sensor that is capable of distributed detection of liquid solvents is presented. Sensor interrogation using Optical Time Domain Reflectometry (OTDR) provides the capability of locating solvent spills to a precision of $\pm 2 \mathrm{~m}$ over a total sensor length that may extend to $20 \mathrm{~km}$.

Introduction: In this Letter an optical fibre sensor capable of making fully distributed measurements of a wide range of solvents is identified. Microbending principles have been used to develop the sensor such that it responds on contact with liquid solvents, typically within 30 seconds of exposure. The sensor incorporates a swelling polymer and optical fibre components that are readily available in the optical communications industry. A conventional optical time domain reflectometry (OTDR) technique is utilised for sensor interrogation and location of multiple spill events over a range of $20 \mathrm{~km}$. The sensor uses a low-power laser source to perform the sensing function and due to its optical nature is intrinsically safe. The operational characteristics of the sensor and the underlying technology utilised in its operation are described. The swelling characteristics of the polymer material in a range of solvents and experimental test results of prototype sensors are presented.

Sensor Design: The sensor structure consists of a central glass reinforced plastic strength member $0.9 \mathrm{~mm}$ in diameter coated with a $100 \mu \mathrm{m}$ thick silicone polymer (Dow Corning Silastic rubber). A graded index multimode optical fibre (Plasma 62.5/125 graded index PCVD fibre) is held against it by helically wrapping a $100 \mu \mathrm{m}$ diameter Kevlar ${ }^{\mathrm{TM}}$ thread along the sensor length as shown in Fig. 1. The silicone polymer swells on contact with the solvents without dissolving; the extent being dependent on the activating solvent and the particular 
silicone polymer used. The swelling induced by the presence of the solvent causes the optical fibre to be forced against the Kevlar thread, thus inducing periodic lateral deformation. The optical fibre then experiences localised periodic microbending, causing light propagating in the fibre to be coupled between the highest guided modes and nearest cladding modes where it is attenuated. The period of the deformation determines the degree of attenuation that occurs. It was found by Fields [1] that maximum mode coupling occurs when the deformation is equal to a specific periodic spacing $\Lambda$, given by Equation 1 .

$$
\Lambda=\frac{2 \pi a}{\sqrt{(2 \Delta)}}
$$

where $a$ is the radius of the fibre core and $\Delta$ is the maximum relative difference between refractive indices of the core and cladding of the fibre. It was observed by Fields [1] and later by Deimeer [2] that a multiple of this period could be used to induce mode coupling due to the equal propagation constants of adjacent modes. For the multimode fibre used in the sensor, $\Lambda$ was calculated at approximately $1 \mathrm{~mm}$, thus it is possible to apply the Kevlar wrap with a pitch of $2,3, \& 4 \mathrm{~mm}$ to cause mode coupling to occur during sensor activation.

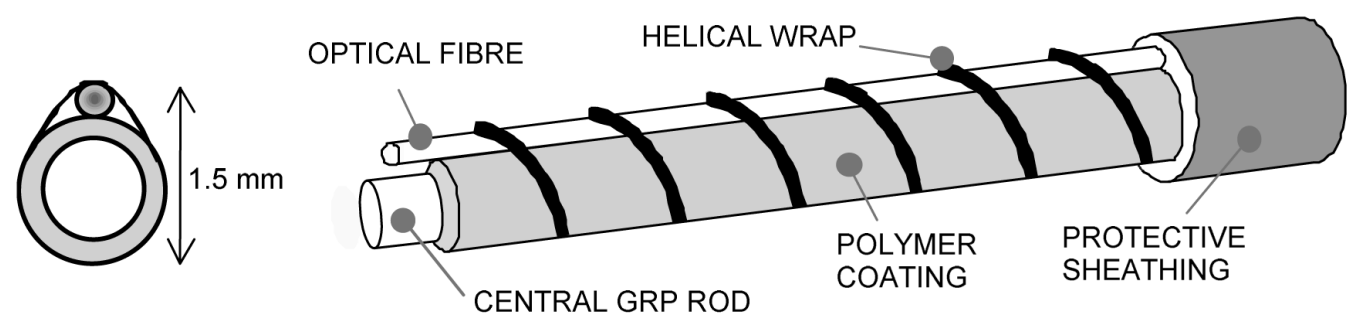

Figure 1 Sensor construction

The sensor is interrogated using standard optical time domain reflectometry (OTDR) principles. OTDR operates by sending a short pulse of light down the optical fibre and monitoring the intensity of the light that is scattered back along the fibre. The OTDR trace displays the single pass loss as a function of distance down the fibre, appearing as a line with 
negative gradient for a fibre with uniform loss. Positions where the loss exceeds the inherent loss of the fibre appear as sections with increased gradient as is shown in Figure 2 schematic.

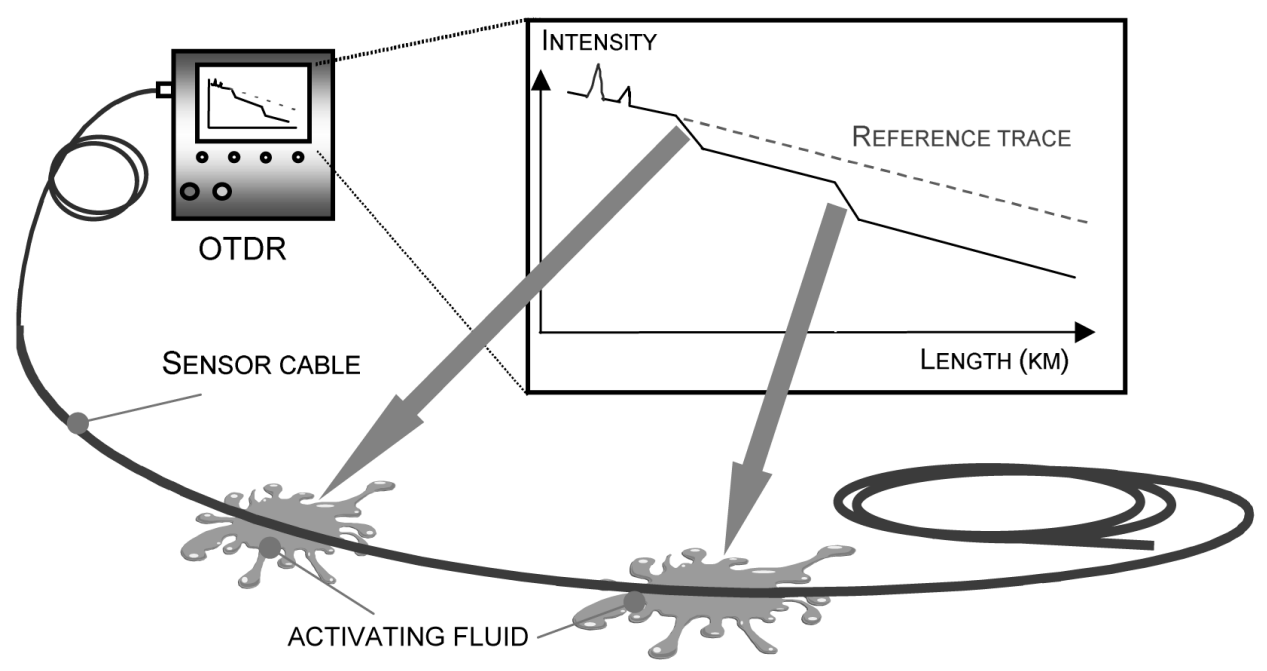

Figure 2 Schematic of sensor operation

Material analysis: In order to determine solvents suitable for detection by the sensor, the silicone used was subjected to a series of tests to estimate the swelling characteristics. It was assumed that the swelling experienced by the silicone could be inferred from the degree of solvent uptake. Small thin samples were weighed when dry and then placed either directly into the solvent or suspended above it in a sealed container. The silicone samples were then removed at regular intervals over a one-hour period to be weighed. It was observed from the experiment that the maximum solvent uptake was achieved typically within 30 seconds when the samples were placed directly into the solvents. Maximum solvent uptake was achieved typically within 1 hour for samples suspended in the solvent vapour. Figure 3 demonstrates the significant difference in solvent uptake for silicone samples placed directly into the solvent and suspended in the vapour. From previous sensor work using swelling hydrogels [3] it was observed that a weight increase of more than $65 \%$ was sufficient to cause a swelling capable of inducing a force on the optical fibre in the sensor. Using this criterion, the experimental results suggested that silicone was suitable for detection of all the solvents 
tested in liquid form, with the exception of acetone. The volatile vapours of chloroform, petroleum ether, cyclohexane, toluene and dichloromethane induced a weight increase that should also be readily detected since the fluid uptake values exceed the $65 \%$ threshold. Neither methyl-ethyl-ketone nor acetone in vapour form induced a solvent uptake value that would be detected using silicone in the current sensor design.

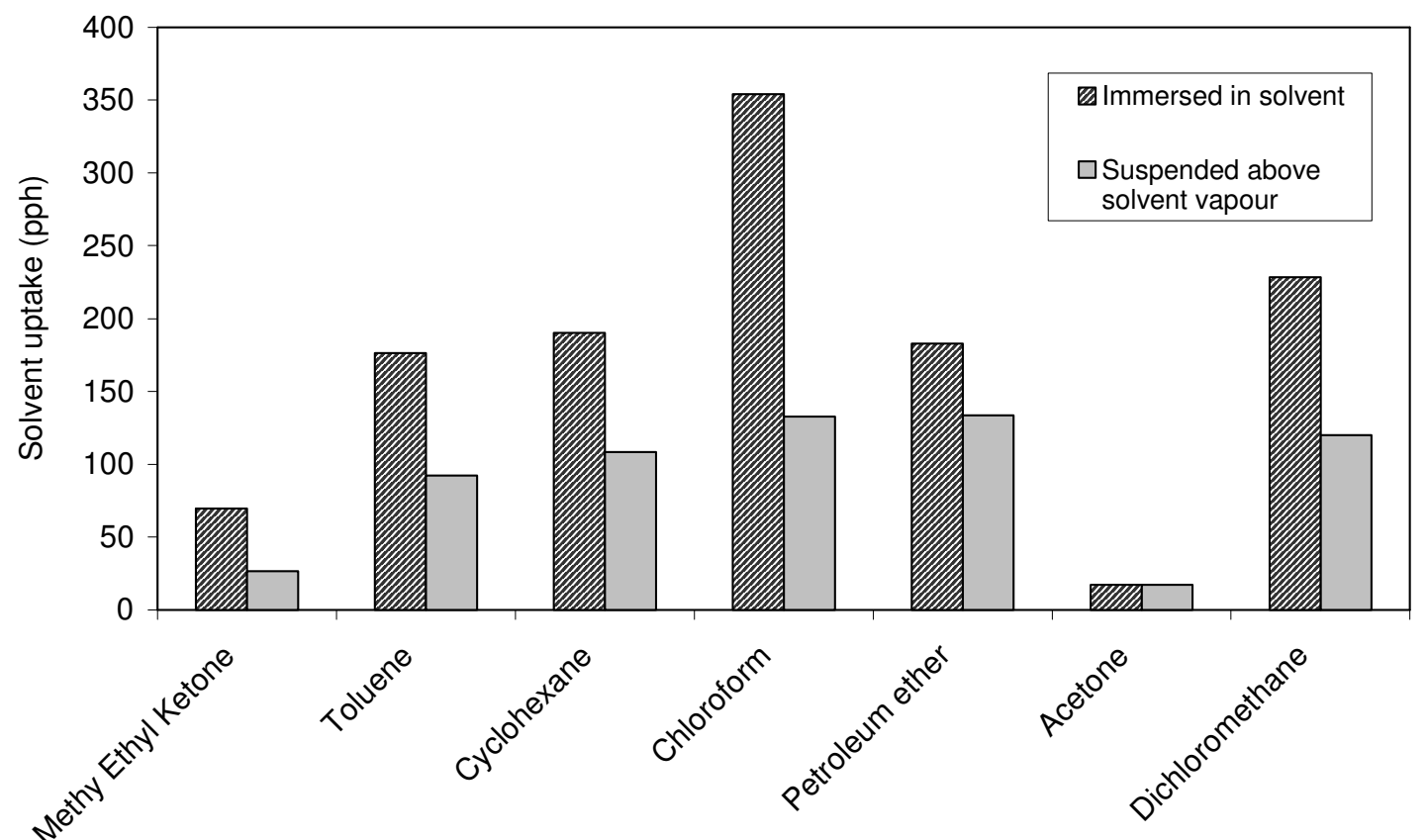

Figure $3 \quad$ Swelling of silicone suspended above and immersed in solvents

Prototype sensor testing: Using the materials knowledge from the above tests, several prototype sensors were manufactured with the assistance of Pinacl Communications using the silicone described in the preceding section and a 62.5/125 graded index multimode fibre. The optical fibre was coated with Hytrel ${ }^{\mathrm{TM}}$, a chemically resistant material incorporated to prevent damage to the outer acrylate layer. The central GRP rod was coated with the silicone to a thickness of $100 \mu \mathrm{m}$ by drawing it through a silicone solution and curing it at an elevated temperature in excess of $150^{\circ} \mathrm{C}$. The Kevlar was wrapped over the coated GRP rod and optical fibre with a pitch of $3 \mathrm{~mm}$, thus easing the precision wrapping requirements during manufacturing. It was observed that the wrapping procedure caused no significant increase in the inherent $3 \mathrm{~dB} / \mathrm{km}$ loss of the fibre due to the buffering effect of the Hytrel. The prototype sensors were evaluated using a commercially available Nortech PC-driven OTDR unit 
operating at an interrogation wavelength of $850 \mathrm{~nm}$ with $20 \mathrm{~ns}$ pulse widths. A $25 \mathrm{~m}$ section of prototype sensor was fusion spliced onto a 50m length of standard optical fibre at either side to eliminate the effects of the near end dead zone and Fresnel reflection at the far end of the fibre. Testing on the prototypes was conducted by capturing a reference OTDR trace of the sensor in its dry state and comparing this with a trace of the sensor experiencing solvent exposure over a 1 metre long section. The loss induced by the swollen silicone when exposed to chloroform induced a $0.12 \mathrm{~dB}$ loss in the optical fibre as shown in Figure 4. The location of the chloroform exposure can be readily identified as the position where the reference and activated traces deviate; corresponding to a position $61 \mathrm{~m}$ along the total fibre length.

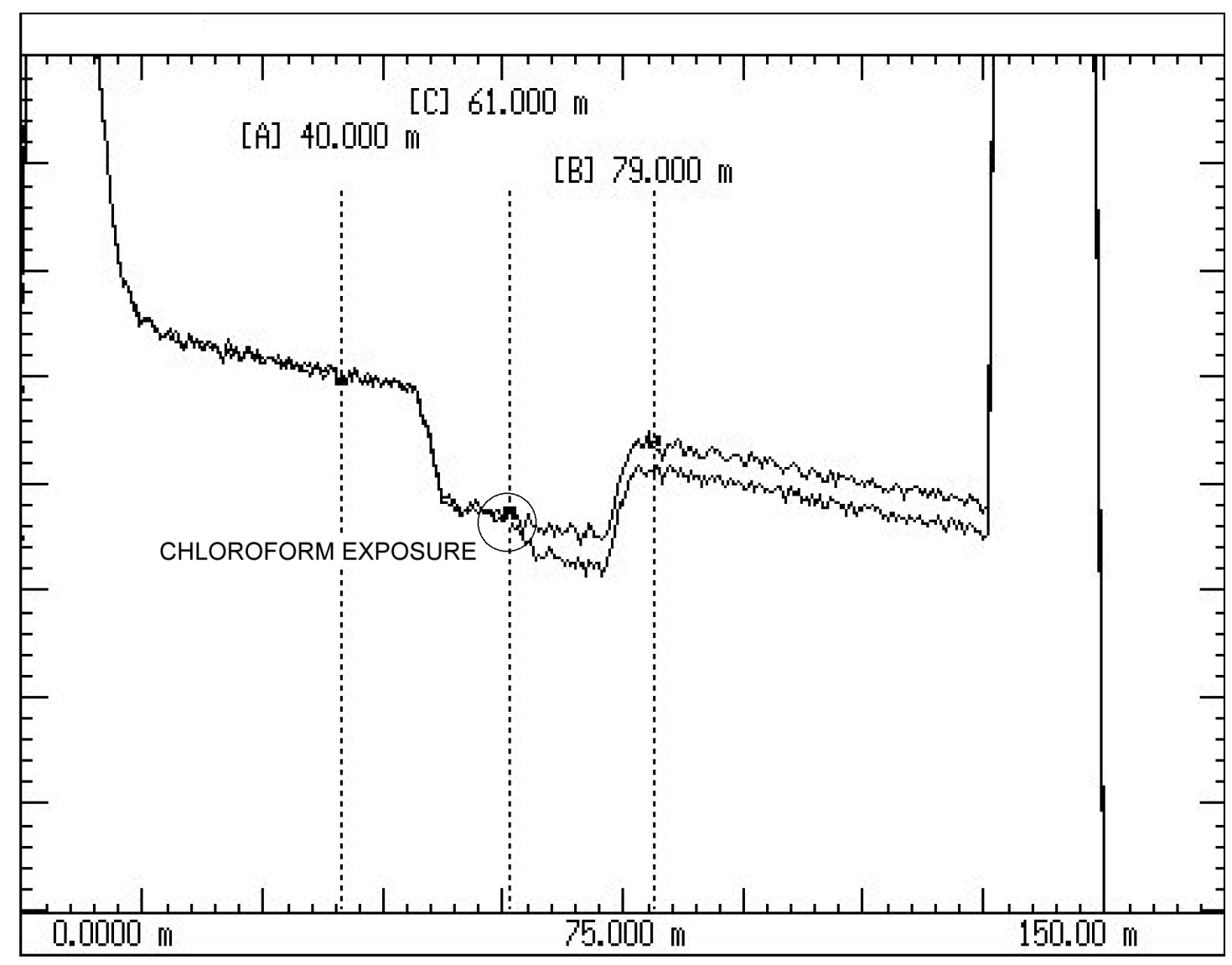

Figure $4 \quad$ OTDR trace of $1 \mathrm{~m}$-long chloroform exposure located $61 \mathrm{~m}$ along the fibre

In addition to the identification of chloroform, the sensor was also utilised to locate exposure to toluene in a manner identical to the initial test. As can be seen from the deviation of the traces in Figure 5, the exposure to toluene caused a loss in the returned power of approximately $0.08 \mathrm{~dB}$ located $58 \mathrm{~m}$ along the fibre length. The resulting loss is lower than 
that induced by chloroform, suggesting that the swelling results shown in Figure 3 are a good indicator of the expected loss induced in the sensor when exposed to a particular solvent. To conclude the tests, the solvents were allowed to fully evaporate from the exposed sensors. When the silicone returned to its dry non-swollen state it was observed that the attenuation of the sensor sections under test had returned to a level similar to the value prior to testing. Subsequent repeat tests demonstrated that it was possible to perform multiple measurements on the same section of exposed sensor without any observed drop in sensitivity.

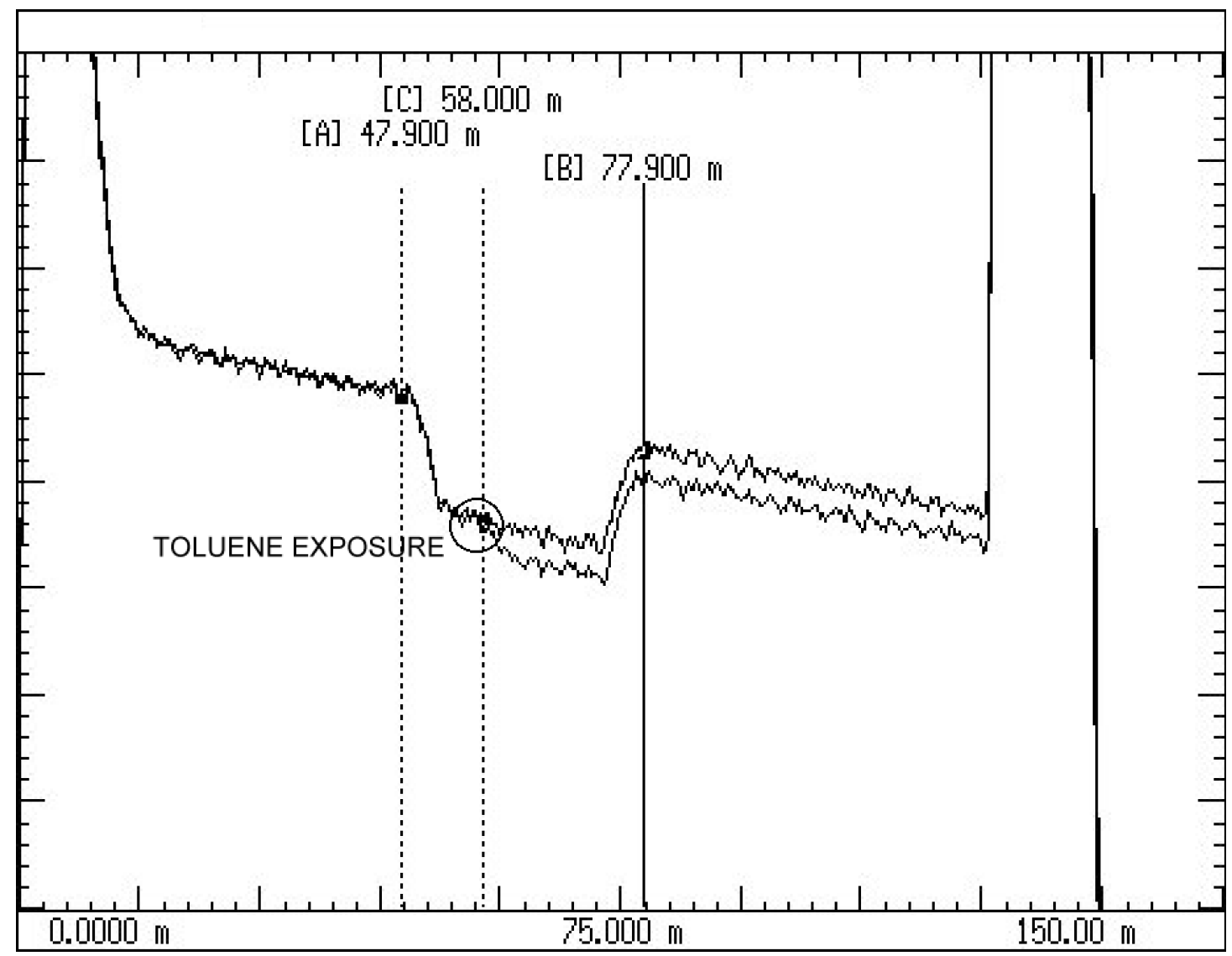

Figure 5 OTDR trace of 1m-long toluene exposure located $58 \mathrm{~m}$ along the fibre

Conclusions: In this paper we have described a fibre optic sensor capable of the distributed detection and location of liquid solvent spills. It has been demonstrated that the sensor is capable of locating the presence of $1 \mathrm{~m}$ lengths of both chloroform and toluene in liquid form due to the swelling of the silicone polymer. Additional fluids or gases may be detected by incorporating an alternative material into the sensor that experiences swelling in their presence. At the OTDR interrogation wavelength of $850 \mathrm{~nm}$, the inherent fibre loss is 
approximately $3 \mathrm{~dB} / \mathrm{km}$, which corresponds to a total sensing range less than $3.5 \mathrm{~km}$ assuming a dynamic range of $20 \mathrm{~dB}$ for the OTDR. It is possible to increase the potential range to $20 \mathrm{~km}$ by shifting to an interrogation wavelength of $1310 \mathrm{~nm}$, where the inherent fibre loss is $0.5 \mathrm{~dB} / \mathrm{km}$, assuming a similar OTDR dynamic range at this wavelength. The sensor is well suited to applications where detection of solvent spills is required over extended areas where point sensors could prove costly. It is particularly suited to environments where there is a high risk of explosion. The distributed measurement capability provides effective monitoring and rapid location of spills in large oil refineries, chemical plants or extensive pipeline networks.

\section{Acknowledgements}

EPSRC and DTI are thanked for their support of this work through the 'DISH' LINK scheme. The contributions of Pinacl Communications, SDA Protec and OptoSci are also gratefully acknowledged.

\section{References}

1. FIELDS J.N.: 'Attenuation of a parabolic index fibre with periodic bends', App. Phys. Lett., 1979, Vol. 36, pp 779-801.

2. DIEMEER M.B.J. and TROMMEL E.S.: 'Fibre optic sensors: Sensitivity as a function of distortion wavelength', Opt. Lett., June 1984, Vol. 9, (6), pp 260-262.

3. MICHIE W.C. et al,: 'Optical fibre grout flow monitor for post tensioned reinforced tendon ducts', Proc. 2nd European Conference on Smart Structures, 1994, SPIE Vol. 2361, pp186189.

\section{Authors affiliations:}

Alistair MacLean, Chris Moran, Walter Johnstone, Brian Culshaw, ( University of Strathclyde, Glasgow G1 1XW, UK.). Corresponding email: a.maclean@eee.strath.ac.uk Dan Marsh, (PINACL Communications, Bodelwyddan, Rhyl, Denbighshire LL18 5TZ, UK) Paul Parker, (SDA Security Systems, Axis 7, Rhodes Way, Watford, Hertfordshire, UK). 